\title{
Finding building shapes that minimize mean trip times
}

\section{R V Johnson}

The paper provides a computer-aided means of ensuring that a proposed multiple-floor building is shaped so that the time that people need to move around inside the building is minimized. The metric that captures this is the mean trip time. This is lower, for example, on a square floor than on an elongated floor of the same area. Spreadsheet formulae are provided to identify the number and shape of building floors that minimize mean trip time given building areas, people's walking speed, and lift speed and wait times. In the case of buildings with two floor sizes, the optimal number of floors of each size and the ratio of the two floor areas are also found. These formulae assume evenly distributed trips, i.e. that trip starts and finishes are equally and independently distributed throughout the building. This alleviates the need to obtain interdepartment-trip volumes and department areas. New simple distance theorems form the basis of the formulae. One odd finding is that, in some situations, a multiple-floor building with two floor sizes has a marginally lower mean trip time than a building of equal area in which all the floors are the same size.

building shape, productivity, design, layout, layout planning

This paper provides a computer-aided method of determining which building shapes are suitable for efficient layouts on the basis of just four readily available items of information. Formulae are provided so that anyone with a basic knowledge of spreadsheets can copy the formulae and use them for their own analyses. These results can be used in a broader analysis, not discussed in this paper, of building shape that includes criteria such as costs of construction, energy and land. The mean trip time can be readily converted to a cost that can be weighed against these criteria.

School of Business Administration, University of Michigan, Ann Arbor, MI 48109-1234, USA

Paper received: 9 May 1991. Accepted: 26 September 1991

\section{PREVIOUS APPROACHES}

A number of computer-aided approaches locate departments within a building of a predetermined shape so the total time or distance of all the trips between departments is minimized. The first approach was that of Armour and Buffa ${ }^{1}$. Their idea was extended to multiple-floor buildings by Cinar ${ }^{2}$, Liggett and Mitchell ${ }^{3}$, Johnson ${ }^{4}$, Kaku, Thompson and Baybars ${ }^{5}$ and others. In all these studies, layout efficiency is captured as the sum of the products of interdepartmental travel times or distances and the number of trips within an arbitrary time interval, or a close approximation. When this is divided by the number of trips, a more readily understood metric is obtained: mean trip distance, or, in the multiple-floor case, mean trip time, which resolves the problem of adding horizontal and vertical distances to time spent waiting for lifts. Some approaches, known as improvement procedures, improve on a provided starting layout, while constructive procedures start from scratch. Many building shapes can be tested with layout methods until a satisfactory shape is found.

\section{ROLE OF NEW APPROACH}

The author's approach does not provide a departmental block layout. Instead, it reveals building shapes that provide the minimum mean trip time under the assumption that trips start and finish independently and uniformly throughout the building. Its great advantage over layout-focused approaches is that it avoids the significant task of collecting data of department areas and the estimated interdepartmental traffic volumes. Further, these traffic patterns may change over time, and so the uniformity assumption may be more robust for the long term.

The author's method has two roles. First, if the department data or the software to perform a layoutbased analysis is unavailable, results derived from the assumption of an even distribution of trips throughout the building are a reasonable substitute. Second, even when department data is available, the computational 


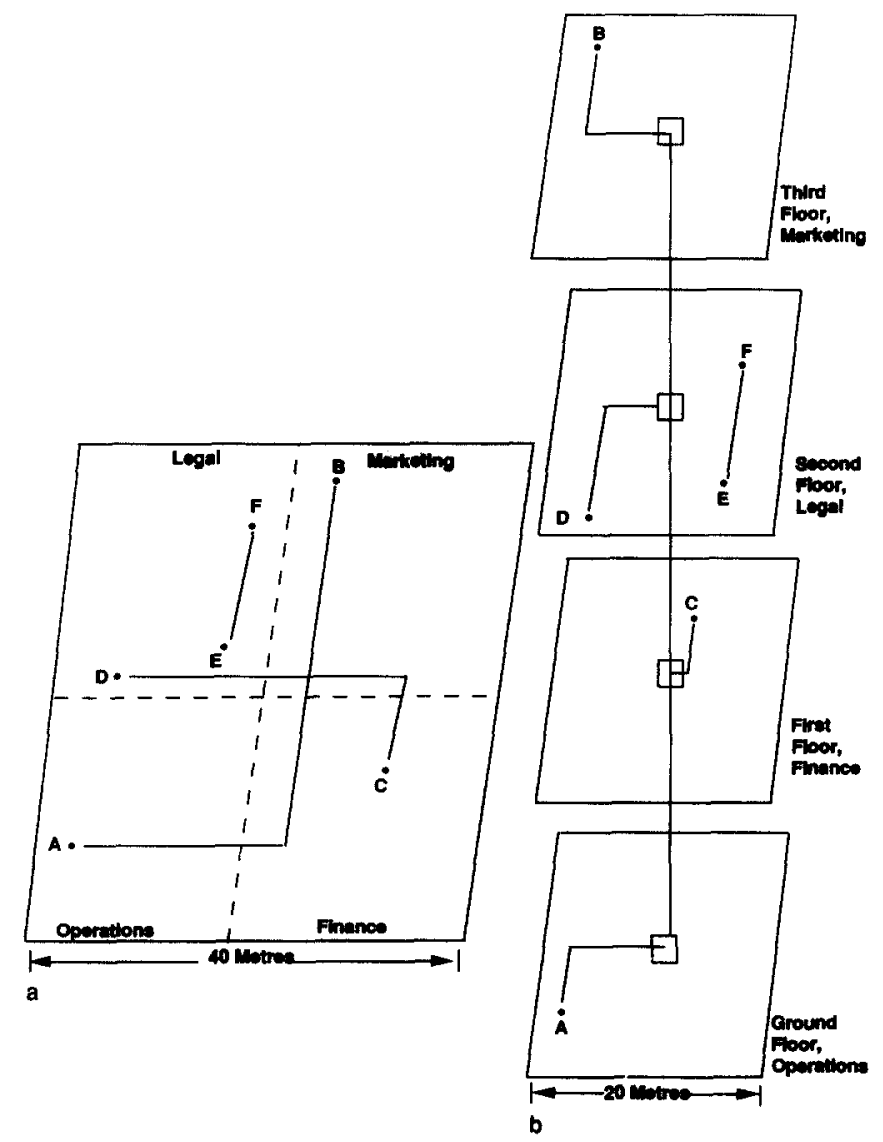

Figure 1. Necessary routes for three sample trips in two buildings of equal area

[Mean trip time for three sample trips $=\left(t_{\mathrm{AB}}+t_{\mathrm{CD}}+t_{\mathrm{EF}}\right) / 3$.

burdens of layout methods are heavy, and the methods described in this paper allow one to obtain a 'big-picture' feel for the tradeoff of floor sizes and the number of floors quite easily.

\section{TYPES OF ORCANIATION WHERE BUILDING SHAPE IS MMPORTANT}

Internal traffic costs are most important in buildings with significant traffic volume. These buildings typically contain just one organization, such as a company head office, a government department, a hospital or an educational institution. Traffic tends to be lighter in buildings with many independent tenants. However, in some buildings with significant traffic, the building shape does not have much impact on the mean trip time. This occurs if a dominant traffic flow exists, as in high-volume dedicated manufacturing. This is unusual in office buildings.

\section{TRIP-DISTANCE THEOREMS}

Theorems and formulae are provided below that relate the mean rectilinear distance of trips to start and finish points that are evenly and independently distributed throughout a building with rectangular floors (see Figure 1). Only one cluster of lifts is considered. (The formulae can be extended for buildings with two or more lift clusters ). Theorem proofs are provided in Appendix 1.

\section{Theorem for single-floor trips}

Theorem 1: For evenly distributed rectilinear trips, the following are true:

- The expected trip distance is one-sixth of the perimeter of the floor.

- A square floor has a lower expected trip distance than any other rectangular floor.

- If the length/width ratio of the floor is $k$, the expected trip distance is greater than that for a square floor of the same area by a factor of $0.5\left(k^{1 / 2}+1 / k^{1 / 2}\right)$, which is called an inefficiency ratio.

A few statistics are computed in Table 1 using the theorems for three sample single-floor building shapes.

\section{Five theorems for mulliple-floor trips}

Theorem 2: If a rectilinear trip originates at an arbitrary point on a rectangular floor and ends at the centroid, the expected trip distance is one-eighth of the floor's perimeter.

This gives the expected distance from an arbitrary point on a floor to lifts located at the centroid.

Theorem 3: If a rectilinear trip originates at an arbitrary point on a rectangular floor and ends at a specific point that is a fraction $x$ of the way along the length $g$ of the floor, and a fraction $y$ of the way along the breadth $b$ of the floor, the expected trip distance is $\left(x^{2}-x+0.5\right) g+$ $\left(y^{2}-y+0.5\right) b$.

This gives the expected distance from an arbitrary point on a floor to a lift located at any fixed point.

Theorem 4: An interfloor trip that originates on the lowest floor and ends on another, arbitrary, floor in a building with $n$ floors travels an expected $n / 2$ floors.

Theorem 5: An interfloor trip that originates and ends on a different, arbitrary, floor of a building with $n$ floors travels an expected $(n+1) / 3$ floors.

Corollary 1: The expected number of floors travelled is $(m+1) / 3$ for trips travelled solely between the lower $m$ floors.

Corollary 2: The expected number of floors travelled is $(n-m+1) / 3$ for trips travelled solely between the upper $(n-m)$ floors.

Table 1. Sample statiatica for three foors of equal area

\begin{tabular}{lccc}
\hline & $\begin{array}{c}\text { Square floor } \\
(4 \times 4)\end{array}$ & $\begin{array}{c}\text { Rectangular floors } \\
(8 \times 2)\end{array}$ & $(16 \times 1)$ \\
\hline Perimeter* & 16.000 & 20.000 & 34.000 \\
Length/width ratio & $1: 1$ & $4: 1$ & $16: 1$ \\
Mean rectilinear trip distance* & 2.660 & 3.330 & 5.660 \\
Inefficiency ratio & 1.000 & 1.250 & 2.125 \\
\hline
\end{tabular}

[* The unit of distance is the length of a module side.] 
Theorem 6: In a building with $n$ floors, the mean number of floors travelled in a trip between one of the lower $m$ floors and one of the upper $(n-m)$ floors is $n / 2$.

Theorem 4 is relevant for trips that originate at a building's ground floor, while Theorems 5 and 6 are relevant for travel within a building. Corollaries 1 and 2 and Theorem 6 are relevant in buildings with two floor sizes, the lower $m$ floors being larger than the upper $(n-m)$ floors.

Important assumptions lie behind these theorems. First, it is assumed that the lift speeds and wait times are targets that are achieved by the selection of the correct number and speeds of lifts. With few floors, a smaller percentage of the trips require a lift than for a building with many floors, and so a relatively lower lift capacity is required. Second, as these formulae assume evenly distributed trips, the interfloor lift times and wait times do not depend on the layout of a particular building shape.

\section{MEAN TRIP-TIME FORMULA}

The mean trip-time formula is derived below for rectilinear trips with evenly distributed and independent start and finish points. The user tests the formula for independent variable values of interest, given the four required data items.

\section{Independent variables:}

number of floors

$$
=n
$$

length $/$ breadth ratio of each floor $=k$

\section{Required data:}

$$
\begin{array}{ll}
\text { horizontal speed of people } & =h \mathrm{~m} / \mathrm{s} \\
\text { vertical time of lifts } & =v \mathrm{~s} / \text { floor moved } \\
\text { expected wait time for lift } & =w \mathrm{~s} \\
\text { building area } & =A \mathrm{~m}^{2}
\end{array}
$$

\section{Mean trip time:}

mean trip time

$$
\begin{aligned}
= & \text { (mean trip time for trips that stay on one floor }) \\
& \times \text { (proportion of trips that stay on one floor }) \\
& +\{\text { (mean trip time for trips going to and } \\
& \quad \text { from lifts }) \\
& +(\text { mean time in a lift }) \\
& + \text { (mean time waiting for a lift })\} \\
& \times \text { (proportion of trips that go to } \\
& \text { another floor }) \\
= & \left\{(A / n)^{1 / 2} / 3\right\}\left(k^{1 / 2}+1 / k^{1 / 2}\right)(1 / h)(1 / n) \\
& +\left\{(A / n)^{1 / 2} / 2\right\}\left(k^{1 / 2}+1 / k^{1 / 2}\right)(1 / h)\{(n-1) / n\}
\end{aligned}
$$

$$
\begin{aligned}
& \times\{v(n+1) / 3\}\{(n-1) / n\} \\
& \times w\{(n-1) / n\}
\end{aligned}
$$

This formula is developed in Appendix 2.

\section{DETERMINATION OF NUMBER OF FLOORS THAT MINIMIZES MEAN TRIP TIME}

The mean trip-time formula should be used for each contending number $n$ of floors. The use of a spreadsheet program makes this a straightforward calculation. The program quickly reveals the optimal number of floors, and it permits sensitivity analysis, as demonstrated for the following public-utility company example.

\section{PUBLIC-UTILITY COMPANY EXAMPLE}

Data was collected so that the building shape that minimized the mean trip time could be found, and so that space could be allocated, for a new head-office building planned for a public-utility company. For this data, the mean trip time was the shortest for a 25 -floor building (see Table 2 ).

$$
\begin{array}{rlrl}
h & =\text { horizontal speed of people } & =0.87 \mathrm{~m} / \mathrm{s} \\
v & =\text { vertical time of lifts } & & =4 \mathrm{~s} / \text { floor moved } \\
w & =\text { wait time for lift } & & =24 \mathrm{~s} \\
A & =\text { building area } & & =60000 \mathrm{~m}^{2}
\end{array}
$$

Fixed decision variable:

$$
k=\text { length } / \text { breadth ratio of each floor }=4
$$

(constrained by real-estate shape).

If the floor shape (represented by the $k$ ratio) is a decision variable rather than a constant, the calculations should be repeated for contending ratios. The second point in Theorem 1 asserts that the lowest mean trip time is obtained with square floors $(k=1)$.

\section{BUILDINGS WITH TWO FLOOR SIZES}

Many multiple-floor buildings have more than one floor size. A building with upper floors that are smaller than the lower floors can have a faster mean trip time than a building of the same area with only one floor size. If the trips are equally distributed, the maximum difference is about $1 \%$. To find some logic in this curious result, consider that the volumetric shape that allows the shortest mean trip time, when the trip is unencumbered by such entities as floors and walls, is a sphere. In a move towards reality via a requirement that the building's shell consist only of horizontal and vertical parts, with all the floors horizontal, and all the vertical travel taking place in a central lift, it may be conjectured that the optimal shape of the building is a series of cylinders, one for each floor, all of which have their center over the same point, but with floor areas that are small on the top and bottom floors, and largest on the middle floor. This shape is as close to a sphere as is practical. If no floor can be 
Table 2. Results of public-utility company example

\begin{tabular}{|c|c|c|c|c|c|}
\hline $\begin{array}{c}\text { Number } \\
\text { of } \\
\text { floors }\end{array}$ & $\begin{array}{l}\text { Expected } \\
\quad \text { total } \\
\text { trip time }\end{array}$ & $\begin{array}{l}\text { Expected } \\
1 \text {-floor } \\
\text { trip time }\end{array}$ & $\begin{array}{l}\text { Expected } \\
\text { time to } \\
\text { and from lift }\end{array}$ & $\begin{array}{l}\text { Expected } \\
\text { time in } \\
\text { lift }\end{array}$ & $\begin{array}{l}\text { Expected } \\
\text { time waiting } \\
\text { for lift }\end{array}$ \\
\hline 1 & 234.63 & 234.63 & 0.00 & 0.00 & 0.00 \\
\hline 2 & 221.38 & 82.95 & 124.43 & 2.00 & 12.00 \\
\hline 3 & 200.17 & 45.15 & 135.46 & 3.56 & 16.00 \\
\hline 4 & 184.30 & 29.33 & 131.98 & 5.00 & 18.00 \\
\hline 5 & 172.50 & 20.99 & 125.91 & 6.40 & 19.20 \\
\hline 10 & 142.38 & 7.42 & 100.16 & 13.20 & 21.60 \\
\hline 20 & 126.78 & 2.62 & 74.76 & 26.60 & 22.80 \\
\hline 22 & 126.08 & 2.27 & 71.62 & 29.27 & 22.91 \\
\hline 24 & 125.79 & 2.00 & 68.85 & 31.94 & 23.00 \\
\hline 25 & 125.77 & 1.88 & 67.57 & 33.28 & 23.04 \\
\hline 26 & 125.83 & 1.77 & 66.37 & 34.62 & 23.08 \\
\hline 28 & 126.15 & 1.58 & 64.13 & 37.29 & 23.14 \\
\hline 30 & 126.70 & 1.43 & 62.11 & 39.96 & 23.20 \\
\hline 40 & 131.88 & 0.93 & 54.26 & 53.30 & 23.40 \\
\hline 50 & 139.60 & 0.66 & 48.78 & 66.64 & 23.52 \\
\hline
\end{tabular}

larger than a lower floor (to keep the construction manageable), and just two rectangular floor sizes are permitted, the permissible shape that is closest to a sphere is the shape described in the second sentence of this paragraph.

A formula is provided for computing the mean trip time within a building with two floor sizes, the smaller floors being at the top.

\section{Decision variables:}

number of floors

$$
=n
$$

number of larger-sized floors

ratio of length to breadth on each floor

$$
=k
$$

ratio of area of larger floor to area of smaller floor $=r$

\section{Required data:}

horizontal speed of people $=h \mathrm{~m} / \mathrm{s}$

vertical time of lifts $\quad=v s /$ floor moved

wait time for lift $\quad=w \mathrm{~s}$

building area $=A \mathrm{~m}^{2}$

Expected time of one trip:

$$
\begin{aligned}
\text { Time }= & {\left[\left\{(A /(m r+n-m))^{1 / 2} / 3\right\}\left(k^{1 / 2}+1 / k^{1 / 2}\right) / h\right] } \\
& \times\left[(n-m) /(m r+n-m)^{2}\right] \\
& +\left[\left\{(r A /(m r+n-m))^{1 / 2} / 3\right\}\right. \\
& \left.\times\left(k^{1 / 2}+1 / k^{1 / 2}\right) / h\right]\left[m r^{2} /(m r+n-m)^{2}\right] \\
& +\left[\left\{(A /(m r+n-m))^{1 / 2} / 2\right\}\right. \\
& \left.\times\left(k^{1 / 2}+1 / k^{1 / 2}\right) / h\right] \\
& \times\left[(n-m)(n-m-1+m r) /(m r+n-m)^{2}\right] \\
& +\left[\left\{(r A /(m r+n-m))^{1 / 2} / 2\right\}\right. \\
& \left.\times\left(k^{1 / 2}+1 / k^{1 / 2}\right) / h\right] \\
& \times\left[m r(m r-r+n-m) /(m r+n-m)^{2}\right] \\
& +(w)\left[\left(1-\left(m r^{2}+n-m\right) /(m r+n-m)^{2}\right)\right]
\end{aligned}
$$

$$
\begin{aligned}
& +[v(n-m+1) / 3] \\
& \times\left[(n-m)(n-m-1) /(m r+n-m)^{2}\right] \\
& +[v(m+1) / 3] \\
& \times\left[\left(m r^{2}(m-1) /(m r+n-m)^{2}\right)\right] \\
& +[v(n / 2)]\left[\left(2 m r(n-m) /(m r+n-m)^{2}\right)\right]
\end{aligned}
$$

This formula is developed in Appendix 3. An implemented spreadsheet, complete with formulae, is shown in Table 3. This spreadsheet can be used for buildings with one floor size by the number of larger-sized floors being set to 0 , or to the total number of floors (i.e. either set $m=0$, or $m=n$ ).

\section{OBTAMMLC DUMmun gung MTH

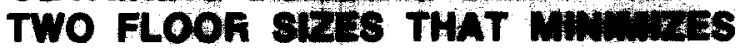 MEAN TRIP TIME}

A spreadsheet should contain the formula for the expected time of one trip, which has been reduced to contain only four decision variables $n, m, k$ and $r$, with four data items $w, v, h$ and $A$. Use a sequential univariate search by varying each variable through all (this is important) of its feasible values to find the shortest mean trip time while holding the other three variables constant. Then, vary the next decision variable. Cycle through the four decision variables repetitively until a cycle is completed in which no improvement in the mean trip time can be found. This obtains a local minimum, which is probably also the global minimum. Two of the variables are integers, and two are real numbers. When the real numbers are varied, a small interval of 0.10 should be used, as the response curve has a diagonal ridge. The use of a larger interval can lead to the ridge leading to the optimum being missed. Once this has been done, it pays to make a further check into a region where the true optimum, if it has been missed so far, always seems to lie. To do this, increase the values of $m$ and $n$ by 1 , and increase $r$ by 0.1 . Then, repeat the search.

To find out how likely this procedure is to find the optimum, an experiment was performed to see if the recommended procedure globally minimized the expected 
Table 3. Spreadsheet and formulae for buildings with two floor sizes

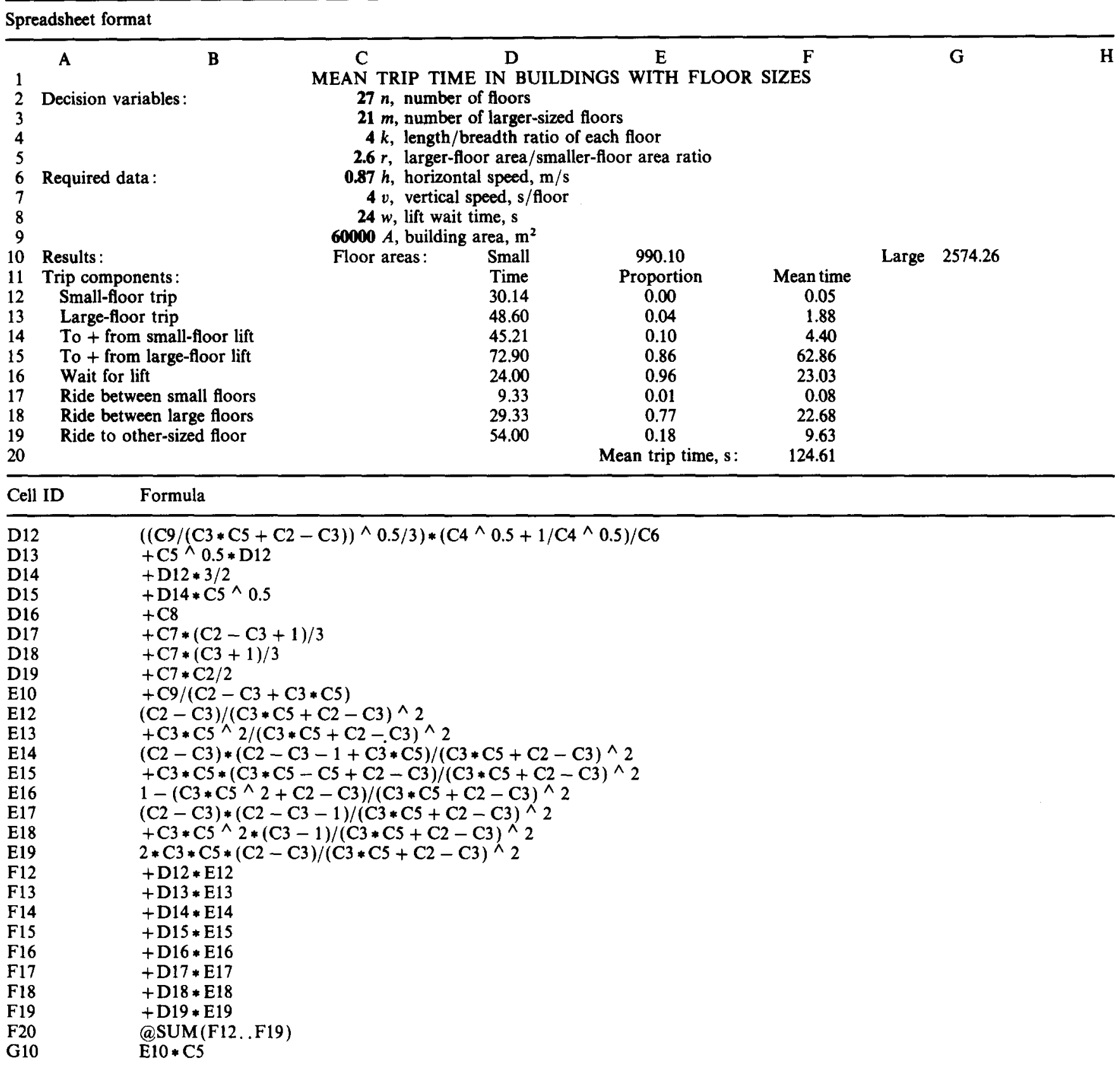

[Items to be input are shown in bold typeface.]

trip time for any specific set of variables required. For each of 43197 problems (different combinations of the four data variables), two searches were conducted to find the best combination of the four decision variables. One was the sequential univariate search described above. The other was an enumerative search that considered 64000 combinations of decision-variables values. Thus, 2764608000 combinations of data variables and decision variables were calculated. Of the 43197 problems, both procedures obtained the optimal mix of decision-variable values for 43100 problems. Of the other 97 problems, the sequential univariate search found a solution that was, at worst, $0.01 \%$ above the global optimum. Also, in all 97 cases, it was observed that, if $n$ and $m$ are increased by 1 , and $r$ is increased by 0.1 , above the local optimal, and a univariate sequential search is resumed from there, a global optimum is found. This justifies the use of the modified univariate search, which has the distinct advantage of being able to be easily and quickly executed on a spreadsheet.

\section{PUBLIC-UTILITY BUILDING: TWO FLOOR SIZES}

For the area, movement times and $k$ ratio of the single floor-size example, the building shape with the lowest mean trip time has 27 floors, 21 being of the larger size, and a ratio of the larger floor area to the smaller floor area of $2.6: 1$. The mean trip time is 124.61 , which is $0.92 \%$ better than can be achieved in a building in which all the floors have the same area. When the number of floors is varied, the mean trip time changes in the 
Table 4. Results of example of public-utility company with two floor sizes

\begin{tabular}{lcl}
\hline $\begin{array}{l}\text { Number of } \\
\text { larger floors }\end{array}$ & Mean trip time & \multicolumn{1}{c}{ Comments } \\
\hline 27 & 125.96 & All floors equally sized \\
25 & 125.20 & \\
24 & 124.94 & \\
22 & 124.64 & Fastest trip \\
21 & 124.61 & \\
20 & 124.67 & \\
15 & 126.16 & \\
10 & 129.40 & \\
5 & 132.35 & \\
4 & 132.38 & Slowest trip \\
3 & 131.97 & All floors equally sized \\
2 & 130.95 & \\
1 & 129.06 & \\
0 & 125.96 & \\
\hline
\end{tabular}

interesting nonlinear way shown in Table 4 for a 27 -floor building with a $k$ ratio of 4 and a ratio of the larger floor area to the smaller floor area of $2.6: 1$.

\section{COMPARISON OF RESULTS OF LAYOUT AND SPREADGHEET APPROACHES}

Does the new spreadsheet method produce a building shape that is similar to that produced by the layout approach? This is a potentially awkward question, as, if the results were different, it would be difficult to determine whether the problem lay in the spreadsheet assumptions or in the effectiveness of the ability to search with the more cumbersome layout methods used for each contending building shape.

In the case of the public-utility building, Johnson's SPACECRAFT $^{4}$, a layout-based approach that was improved by an unpublished layout-generating procedure that made it a constructive, followed by an improvement, procedure, identified that 25 floors were best when all the floors were the same size. The same conclusion was drawn by the spreadsheet method. When two floor sizes were permitted, the results of the two procedures were in the same ballpark, but were not precisely the same. Of the 21 building configurations examined by the layout-based method, the best one according to the formulae was the seventh best according to the layout method. The best according to the layout method was the second best according to the formulae.

This result indicates that interdepartment traffic patterns have some impact on the optimal building shape when two floor sizes are permitted. Otherwise, the spreadsheet and the layout-based method arrive at the same optimum. In the presence of a specific and lasting traffic pattern, a shortening of the mean trip time may be achieved by the careful selection of the building shape and the careful determination of the layout of departments. To obtain a saving, the recommended procedure is to reach the ballpark of best configurations by the use of the spreadsheet formula, and to refine the building shape by the inputting of traffic-volume data into a layout-based constructive layout generator. If only one floor size is contemplated, the spreadsheet formulae are likely to give sufficiently accurate results. Either approach is likely to derive a considerably more efficient building than that obtained if the question of internal traffic is ignored.

\section{FUTURE RESEARCH: SHAPING BUILDINGS WITH MULTIPLE CRITERIA}

Considerable analysis and judgement is required in the blending of the several criteria relevant to the shaping of a building. Immediate costs include real-estate and construction costs, and ongoing costs include energy and traffic costs, and property taxes. Uncertainty of future use is an issue. In case the building is to be sold or leased some day, it should be located and sized in a way that is in demand. The patterns of use of most buildings change over time. A further difficulty in relation to the buildingshape decision is that of obtaining enough information. The estimation of energy and construction costs for contending building shapes is time-consuming and expensive. Also, as the analysis becomes more careful, so does the need for data accuracy. At what point should the search for a better configuration stop? The shaping of buildings is an art that can utilize many scientific results, including the results offered in this paper. More research is needed for the shaping of buildings to become a science.

\section{REFERENCES}

1 Armour, G C and Bufia, E S 'A heuristic algorithm and simulation approach to relative location of facilities' Manage. Sci. Vol 9 No 1 (1963) pp 294-309

2 Cinar, $U$ 'Facilities planning: a systems analysis and space allocation approach' in Eastman, C (Ed.) Spatial Synthesis in Computer-Aided Building Design John Wiley, USA (1975) pp 19-40

3 Liggett, $\mathbf{R} \mathbf{S}$ and Mitchell, W $\mathbf{J}$ 'Optimal space planning in practice' Comput.-Aided Des. Vol 13 No 5 (1981) pp 277-288

4 Johnson, $\mathbf{R} \mathbf{V}$ 'SPACECRAFT for multi-floor layout planning' Manage. Sci. Vol 28 No 4 (1982) pp 407-417

5 Kaku, B K, Thompson, G L and Baybars, I 'A heuristic method for the multi-story facilities layout problem' Europ. J. Oper. Res. Vol 37 No 3 (1988) pp 384-397

\section{BIBLIOCRAPHY}

Vollman, T E and Bufn, ES 'The facilities layout problem in perspective' Manage. Sci. Vol 12 No 10 (1966) pp 450-468

\section{APPENDIX 1}

\section{Trip-diatanee thoorem proole}

Theorem 1 (first point): First, it is proven that the expected distance of a trip with random start and finish points within a unit length is one-third of the length. Let 
$x$ and $y$ be random points on a straight line between 0 and 1 , with both points following independent uniform distributions. By symmetry, $P(x>y)=0.5$.

$$
\begin{aligned}
& E(x-y \mid x>y)= \int_{0}^{1} \int_{0}^{x}((x-y) p(x) p(y) \mathrm{d} y \mathrm{~d} x) \\
& P(x>y) \\
&=\int_{0}^{1} \int_{0}^{x}(x-y) \mathrm{d} y \mathrm{~d} x / 0.5 \\
&(\text { as } p(x)=p(y)=1) \\
&= \int_{0}^{1}\left(x^{2} / 2\right) \mathrm{d} x / 0.5 \\
&=(1 / 6) / 0.5 \\
&=1 / 3
\end{aligned}
$$

As rectilinear distances can be split into length and breadth components, the components can be added. The above proof applies to length and breadth distances, proving that the expected trip distance is one-sixth of the perimeter.

Theorem 1 (second point): Let the floor's length be $g$ and its breadth be $b$. The area $A=g b$, and the mean trip distance $=(g+b) / 3=[(g+A / g) / 3]$ and $\mathrm{d} / \mathrm{d} g\{(g+$ $A / g) / 3\}=1 / 3-A g^{-2} / 3 . \mathrm{d} / \mathrm{d} g=0$ being set to find an extreme point, $g=A^{1 / 2}$. This implies that $b=A^{1 / 2}$, proving the second point of Theorem 1.

Theorem 1 (third point): Let the floor's length be $g$ and its breadth be $b$. The area $A=g b$. Also, $k=g / b$. These being solved, $g=(A k)^{1 / 2}$ and $b=(A / k)^{1 / 2}$. The application of these to the second point of Theorem 1 results in an expected trip distance of $\left((A k)^{1 / 2}+(A / k)^{1 / 2}\right) / 3=$ $\left(A^{1 / 2} / 3\right)\left(k^{1 / 2}+1 / k^{1 / 2}\right)$. On a square floor, $k=1$. The inefficiency ratio is therefore $\left(A^{1 / 2} / 3\right)\left(k^{1 / 2}+1 / k^{1 / 2}\right) /$ $\left[\left(A^{1 / 2} / 3\right)(1+1)\right]=0.5\left(k^{1 / 2}+1 / k^{1 / 2}\right)$, proving the third point of Theorem 1 .

Theorem 2: Let the floor's length be $g$ and its breadth be $b$. The mean lengthwise trip distance is half of the distance from the centroid to the end of the row, or $g / 4$. Similarly, the breadthwise distance is $b / 4$. For rectilinear movement, summing obtains the desired result.

Theorem 3: Let the floor's length be $g$ and its breadth be $b$. The area $A=g b$. The rectilinear distance is split into lengthwise and breadthwise components. Consider lengthwise movement, where the fixed point is at $x g$. The proportion of trips that originate at a coordinate lower than $x g$ is $x$, and the mean distance is $x g / 2$. Therefore, the expected distance is $x^{2} g / 2$. The proportion of trips that originate at a coordinate greater than $x g$ is $(1-x)$, and the mean distance is $(1-x) g / 2$. Therefore, the expected distance is $(1-x)^{2} g / 2$. The expected lengthwise distance overall is $x^{2} g / 2+(1-x)^{2} g / 2=$ $\left(x^{2}-x+0.5\right) g$. By similar reasoning, the expected breadthwise movement is $\left(y^{2}-y+0.5\right) b$.
Theorem 4: Trips from the lowest floor (call this Floor 1) to Floors $2,3, \ldots, n$ cover $1,2, \ldots,(n-1)$ floors, respectively. The expected number of floors travelled is therefore $(1+2+\ldots+(n-1)) /(n-1)=(n-1)(n /$ $2) /(n-1)=n / 2$, proving Theorem 4 .

Theorem 5: The proof of Theorem 5 is carried out by mathematical induction. For $n=2$, the mean trip distance is one floor, as this is the only possible trip distance. This is predicted by the formula, as $(2+1) / 3=1$. Assume that the mean trip distance in a building with $n$ floors is $(n+1) / 3$ floors. If one floor is added, the mean distance from the $(n+1)$ th floor to other floors is $(n+1) / 2$. There are $n(n-1) / 2$ routes between $n$ floors. Therefore, the mean over all $(n+1)$ floors is $\{(n(n-1) / 2)$ $(n+1) / 3+n(n+1) / 2\} /\{(n+1)(n / 2)\}=(n+2) / 3$, proving the formula for $(n+1)$ floors. This completes the proof of Theorem 5 .

Corollary 1: Corollary 1 is proved by the application of Theorem 5 to the $m$ larger floors.

Corollary 2: Corollary 2 is proved by the application of Theorem 5 to the $(n-m)$ smaller floors.

Theorem 6: Under the constraint $m=1$, the proof of Theorem 6 is complete by the application of Theorem 4 . Next, it is shown by mathematical induction that the formula applies for any value of $n, 1<m \leqslant n$. The total number of trips is counted, with there being one trip between each pair of floors. Double counting is avoided by the consideration only of trips from the smaller floors to the larger floors. It is assumed that the formula is true for $m$ larger floors. The number of larger floors is increased to $m+1$ by conversion of the lowest smaller floor to the highest larger floor. This has three effects: (a) the total number of trips between floors of different sizes changes from $m(n-m)$ to $(m+1)(n-m-1)$, (b) the reduced total of floors travelled between the converted floor and larger floors is $1+2+\ldots+m=m(m+1) / 2$, and (c) the increased total of floors travelled between the converted floor and smaller floors is $1+2+\ldots+$ $(n-m-1)=(n-m-1)(n-m) / 2$. With $m$ large floors, the total floors moved $=(n / 2)(m)(n-m)$. With $(m+1)$ large floors, the total floors moved $=(n / 2)(m)$ $(n-m)-m(m+1) / 2+(n-m-1)(n-m) / 2=(n / 2)$ $(m+1)(n-m-1)$. From this, the mean number of floors in a trip are computed by $(n / 2)(m+1)$ $(n-m-1) /[(m+1)(n-m-1)]=(n / 2)$, proving the formula for $m+1$ larger floors, and hence proving Theorem 6 .

\section{APPENDIX 2}

\section{Development of mean trip-time formula: single floor size}

Derived expressions

area of each floor $\quad=A / n$ 
$R \vee$ Johnson

length $g$ of each floor $=(A k / n)^{1 / 2}$

breadth $b$ of each floor $=(A / k n)^{1 / 2}$

proportion of trips that go to another floor

$$
=(n-1) / n
$$

proportion of trips that stay on one floor

$$
=1 / n
$$

mean distance of a trip that stays on one floor

$$
=(g+b) / 3
$$

(from Theorem 1, first point)

$$
=\left\{(A / n)^{1 / 2} / 3\right\}\left(k^{1 / 2}+1 / k^{1 / 2}\right)
$$

(from Theorem 1, third point)

mean time of a trip that stays on one floor

$$
\begin{aligned}
= & \left\{(A / n)^{1 / 2} / 3\right\} \\
& \times\left(k^{1 / 2}+1 / k^{1 / 2}\right)(1 / h)
\end{aligned}
$$

mean distance to lifts $=(g+b) / 4$ (from Theorem 2)

$$
=\left\{(A / n)^{1 / 2} / 4\right\}\left(k^{1 / 2}+1 / k^{1 / 2}\right)
$$

(from Theorem 1, third point)

mean time going to and from lifts

$$
\begin{aligned}
= & \left\{(A / n)^{1 / 2} / 2\right\} \\
& \times\left(k^{1 / 2}+1 / k^{1 / 2}\right)\{1 / h\}
\end{aligned}
$$

mean time in a lift $\quad=v(n+1) / 3$ (from Theorem 5) mean time waiting for a lift

$$
=w
$$

\section{Moan trip time}

Mean trip time $=($ mean trip time of trip that stays on one floor)

$x$ (proportion of trips that stay on one floor)

$+\{$ (mean trip time of trip going to and from lifts )

$+($ mean time in a lift)

$+($ mean time waiting for a lift $)\}$

$\times$ (proportion of trips that go to another floor)

$$
\begin{aligned}
= & \left\{(A / n)^{1 / 2} / 3\right\}\left(k^{1 / 2}+1 / k^{1 / 2}\right)(1 / h)(1 / n) \\
& +\left\{(A / n)^{1 / 2} / 2\right\} \\
& \times\left(k^{1 / 2}+1 / k^{1 / 2}\right)(1 / h)\{(n-1) / n\} \\
& +\{v(n+1) / 3\}\{(n-1) / n\} \\
& +w\{(n-1) / n\}
\end{aligned}
$$

\section{APPENDIX 3}

\section{Development of mean trip-time formula: two floor sizes}

\section{Derived expressions}

area of each smaller floor

$$
=A /(m r+n-m)
$$

area of each larger floor

$$
=r A /(m r+n-m)
$$

$g=$ length of smaller floor

$=(A k /(m r+n-m))^{1 / 2}$

$$
\text { (as } g b=a /(m r+n-m) \text { and } k=g / b \text { ) }
$$

$b=$ breadth of smaller floor

$=(A /(k(m r+n-m)))^{1 / 2}$

length of larger floor

$$
\begin{aligned}
& =g r^{1 / 2} \\
& =(r A k /(m r+n-m))^{1 / 2}
\end{aligned}
$$

breadth of larger floor

$$
\begin{aligned}
& =b r^{1 / 2} \\
& =(r A /(k(m r+n-m)))^{1 / 2}
\end{aligned}
$$

$t_{1}=$ mean time of trip that stays on smaller floor

$$
\begin{aligned}
& =(g+b) /(3 h) \quad \text { (Theorem } 1, \text { first point }) \\
& =\left\{(A /(m r+n-m))^{1 / 2} / 3\right\}\left(k^{1 / 2}+1 / k^{1 / 2}\right) / h
\end{aligned}
$$

$t_{2}=$ mean time of trip that stays on larger floor

$$
=\left\{(r A /(m r+n-m))^{1 / 2} / 3\right\}\left(k^{1 / 2}+1 / k^{1 / 2}\right) / h
$$

$t_{3}=$ mean time of trip going to and from lift on smaller floor

$$
\begin{aligned}
& =2(g+b) /(4 h) \quad(\text { Theorem } 2) \\
& =\left\{(A /(m r+n-m))^{1 / 2} / 2\right\}\left(k^{1 / 2}+1 / k^{1 / 2}\right) / h
\end{aligned}
$$

$t_{4}=$ mean time of trip going to and from lift on larger floor

$$
=\left\{(r A /(m r+n-m))^{1 / 2} / 2\right\}\left(k^{1 / 2}+1 / k^{1 / 2}\right) / h
$$

$t_{5}=$ mean time waiting for a lift

$$
=w
$$

$t_{6}=$ mean time in lift on trip between smaller floors

$$
=v(n-m+1) / 3 \quad \text { (Theorem 6) }
$$

$t_{7}=$ mean time in lift on trip between larger floors

$$
=v(m+1) / 3 \quad(\text { Theorem 6) }
$$

$t_{8}=$ mean time in lift on trip between floors of different size

$=v(n / 2) \quad($ Theorem 6)

proportion of trips that start on each smaller floor

$$
=1 /(m r+n-m)
$$


proportion of trips that start on each larger floor

$$
=r /(m r+n-m)
$$

$p_{1}=$ proportion of trips that stay on one of the $(n-m)$ smaller floors

$$
=(n-m) /(m r+n-m)^{2}
$$

$p_{2}=$ proportion of trips that stay on one of the $m$ larger floors

$$
=m r^{2} /(m r+n-m)^{2}
$$

$p_{3}=$ proportion of trips from smaller floor to any other floor

$$
=(n-m)(n-m-1+m r) /(m r+n-m)^{2}
$$

$p_{4}=$ proportion of trips from larger floor to any other floor

$$
=m r(m r-r+n-m) /(m r+n-m)^{2}
$$

proportion of trips that stay on one floor

$$
\begin{aligned}
& =(n-m) /(m r+n-m)^{2}+m r^{2} /(m r+n-m)^{2} \\
& =\left(m r^{2}+n-m\right) /(m r+n-m)^{2}
\end{aligned}
$$

$p_{5}=$ proportion of trips that go to another floor

$$
=1-\left(m r^{2}+n-m\right) /(m r+n-m)^{2}
$$

$p_{6} \approx$ proportion of trips that start on smaller floor and end on another smaller floor

$$
=(n-m)(n-m-1) /(m r+n-m)^{2}
$$

$p_{7}=$ proportion of trips that start on larger floor and end on another larger floor

$$
=m r^{2}(m-1) /(m r+n-m)^{2}
$$

$p_{8}=$ proportion of trips that are between floors of a different size

$$
=2 m r(n-m) /(m r+n-m)^{2}
$$

\section{Expected time of one trip}

time $=t_{1} p_{1}+t_{2} p_{2}$

(trips within one floor)

$$
\begin{aligned}
& +t_{3} p_{3}+t_{4} p_{4} \quad \text { (to and from lifts) } \\
& +t_{5} p_{5} \quad \text { (waiting for lift) } \\
& +t_{6} p_{6}+t_{7} p_{7}+t_{8} p_{8} \quad \text { (riding lift) } \\
& =\left[\left\{(A /(m r+n-m))^{1 / 2} / 3\right\}\left(k^{1 / 2}+1 / k^{1 / 2}\right) / h\right] \\
& \times\left[(n-m) /(m r+n-m)^{2}\right] \\
& +\left[\left\{(r A /(m r+n-m))^{1 / 2} / 3\right\}\left(k^{1 / 2}+1 / k^{1 / 2}\right) / h\right] \\
& \times\left[m r^{2} /(m r+n-m)^{2}\right] \\
& +\left[\left\{(A /(m r+n-m))^{1 / 2} / 2\right\}\left(k^{1 / 2}+1 / k^{1 / 2}\right) / h\right] \\
& \times\left[(n-m)(n-m-1+m r) /(m r+n-m)^{2}\right] \\
& +\left[\left\{(r A /(m r+n-m))^{1 / 2} / 2\right\}\left(k^{1 / 2}+1 / k^{1 / 2}\right) / h\right] \\
& \times\left[m r(m r-r+n-m) /(m r+n-m)^{2}\right] \\
& +(w)\left[\left(1-\left(m r^{2}+n-m\right) /(m r+n-m)^{2}\right)\right] \\
& +[v(n-m+1) / 3] \\
& \times\left[(n-m)(n-m-1) /(m r+n-m)^{2}\right] \\
& +[v(m+1) / 3]\left[\left(m r^{2}(m-1) /(m r+n-m)^{2}\right)\right] \\
& +[v(n / 2)]\left[\left(2 m r(n-m) /(m r+n-m)^{2}\right)\right]
\end{aligned}
$$

Roger $V$ Johnson is an associate professor of operations management at the University of Michigan School of Business Administration, USA, where he teaches MBA Operations Management classes. He is the Academic Director of the school's Executive Education courses in project management and strategic quality management. His principle research

interests are assembly-line balancing and management, project management, facility layout and building shape, and quality management. He gained his doctorate at the University of California at Los Angeles, USA. Prior to moving to Michigan, he served as Dean of Commerce at the University of Otago in New Zealand and as a visiting associate professor at UCLA. Professor Johnson has published in various journals. 